\title{
Effects of backrest positioning and gear ratio on nondisabled subjects' handcycling sprinting performance and kinematics
}

\author{
Arnaud Faupin, PhD; ${ }^{1-2 *}$ Philippe Gorce, $\mathrm{PhD} ;{ }^{3}$ Christophe Meyer, $\mathrm{MS}^{\mathbf{4}}$ André Thevenon, $\mathrm{MD}, \mathrm{PhD}^{\mathbf{1 , 5}}$ \\ ${ }^{1}$ Laboratoire d'Etudes de la Motricité Humaine, Faculté des Sciences du Sport et de l'Education Physique, \\ Université de Lille 2, Ronchin, France; ${ }^{2}$ Laboratoire d'Automatique de Mécanique et d'informatique Industrielles et \\ Humaines, Université de Valenciennes, Valenciennes, France; ${ }^{3}$ Laboratoire Ergonomie Sportive et Performance, \\ Université du Sud Toulon-Var, La Garde, France; ${ }^{4}$ Department of Rehabilitation Sciences, Katholieke Universiteit \\ Leuven, Leuven, Belgium; ${ }^{5}$ Centre Hospitalier Régional Universitaire de Lille, Service Médecine Physique et \\ Réadaptation, Lille, France
}

\begin{abstract}
Backrest position is a significant parameter in handcycling that one must consider when seeking to optimize the user-to-chair interface. We studied the effects of backrest position on handcycle propulsion kinematics. Ten nondisabled participants with no handcycle propulsion experience repeated an $8 \mathrm{~s}$ sprint with three backrest positions (backrest angle between $45^{\circ}$ and $50^{\circ}$ to the horizontal, backrest angle between $65^{\circ}$ and $70^{\circ}$ to the horizontal, and without backrest) and three gear ratios (GRs) (GR22/21 = low, GR32/21 = medium, and GR44/21 = high). We used three-dimensional movement analysis to calculate the average maximal velocity, cycle frequency, and angle parameters for the arms and trunk. Our results showed statistically higher trunk flexion/extension $(p<0.001)$ and maximal velocity $(p<0.001)$ when a backrest was not used. Moreover, these differences were accentuated as the GR increased (GR44/21). Our results suggest that handcycle users with unimpaired upper-body and trunk function can improve handcycling performance by removing the backrest. Nevertheless, future studies on specific groups of subjects with spinal cord injury should be conducted.
\end{abstract}

Key words: backrest, gear ratio, handcycle, kinematics, performance, rehabilitation, spinal cord injury, sports, trunk, wheelchair.

\section{INTRODUCTION}

Over the last 20 years, researchers have shown new interest in a complementary mode of propulsion for wheelchair-dependent users: "handbiking" or "handcycling," which is now widely used in rehabilitation and sporting programs for people with disabilities or reduced mobility. In fact, handcycling became an International Paralympic Committee event for the first time in 2004. A number of studies have shown that this mode of propulsion is more efficient (in terms of mechanical efficiency and cardiorespiratory responses) than the hand rim wheelchair [1-2].

However, research on handcycling is often limited to studies evaluating the physiological responses [1,3-4]. Although the biomechanics of hand rim wheelchair

\footnotetext{
Abbreviations: $\mathrm{Ad} / \mathrm{Ab}=$ adduction/abduction, $\mathrm{ANOVA}$ = analysis of variance, $\mathrm{AP}=$ arm power, $\mathrm{ATP}=$ arm and trunk power, B45 = backrest angle adjusted to between $45^{\circ}$ and $50^{\circ}$ to horizontal, B65 = backrest angle adjusted to between $65^{\circ}$ and $70^{\circ}$ to horizontal, C7 = seventh cervical (vertebra), F/E = flexion/extension, $\mathrm{GR}=$ gear ratio, $\mathrm{L3}=$ third lumbar (vertebra), $\mathrm{Ri} / \mathrm{Re}=$ internal/ external rotation, $\mathrm{ROM}=$ range of motion, $\mathrm{SCI}=$ spinal cord injury, SD = standard deviation, WB = without backrest.

* Address all correspondence to Arnaud Faupin, PhD; Laboratoire d'Etudes de la Motricité Humaine, EA 3608, Université de Lille 2, Faculté des Sciences du Sport et de l'Education Physique (FSSEP), 9 rue de l'Université, F-59790 Ronchin, France; +33-320-887-369; fax: +33-320-887-363.

E-mail: faupin.arnaud@aliceadsl.fr

DOI: 10.1682/JRRD.2006.10.0139
} 
propulsion have often been analyzed [5], few studies have been published on the biomechanics of handcycle propulsion [6-8]. At present, data concerning the kinematic [9], kinetic [10], and surface electromyographic [11] parameters of handcycle propulsion are scarce. Understanding the biomechanics of handcycle propulsion is important for optimizing the performance and ergonomics of the handcycle-user interface.

To the best of our knowledge and despite that much research has been focused on synchronous versus asynchronous crank modes [12-14], few studies have examined the effects of backrest positions, with the exception of van Breukelen [15]. However, van Breukelen merely proposed a theoretical classification of synchronous handbiking according to the backrest position but did not perform any measurements. According to van Breukelen, two means of handcycle propulsion exist: an arm power (AP) only mode and a combined arm and trunk power (ATP) mode [15]. Hence, attachable handcycles can be classified as AP or ATP based on the backrest position and the crank position. Moreover, van der Woude et al. hypothesize in their review that the performance benefits of the synchronous arm mode may also be due to use of the trunk's larger effective muscle mass and weight in propulsion [6]. If the trunk is properly controlled, its flexors and extensors will actively contribute to power production.

Hence, the main objective of the present study was to assess the effect of backrest positions (both alone and combined with different gear ratios [GRs]) on trunk flexion/extension (F/E) during maximal synchronous handcycling sprinting on an ergometer. Although handcyling is mainly an endurance discipline, the handcyclist must also be able to sprint, or promptly speed up from an already high velocity. These actions should require larger muscle mass contributions from the trunk when possible. We consequently hypothesize that changes in backrest position (without a backrest; i.e., ATP type) combined with different GRs could improve performance by increasing trunk movement during the propulsive phase, allowing greater velocity.

\section{METHODS}

\section{Subjects}

Ten nondisabled subjects with no handcycling experience (six men and four women, mean \pm standard deviation $[\mathrm{SD}]$ age $=24.7 \pm 1.3 \mathrm{yr}$, mean \pm SD body mass $=71.5 \pm$
$13.3 \mathrm{~kg}$, mean \pm SD height $=1.77 \pm 0.75 \mathrm{~m}$ ) participated in the study. The present study did not use invasive techniques that could have influenced the participants' well-being or behavior or the biomechanical characteristics of handcycle propulsion. All procedures were fully explained to the participants, who gave their written informed consent for participation. The study was designed in accordance with the ethical standards of the Helsinki Declaration of 1975 and received the approval of the local Consultative Committee for the Protection of Persons in Biomedical Research.

\section{Experimental Protocol}

Once seated in the handcycle, the participants completed a 10 min familiarization session with the equipment. This session also served as a warm-up during which the participants cranked at submaximal intensity to acclimate to the handcycling task and the laboratory environment. At the experimenter's signal, each participant performed a maximal sprint for $8 \mathrm{~s}$, with encouragement from the experimenter.

This $8 \mathrm{~s}$ sprint was performed with three different GRs (GR22/21 = low, GR32/21 = medium, and GR44/21 = high) and three different backrest positions (B45 = backrest angle adjusted to between $45^{\circ}$ and $50^{\circ}$ to horizontal, B65 = backrest angle adjusted to between $65^{\circ}$ and $70^{\circ}$ to horizontal, and $\mathrm{WB}=$ without a backrest), meaning that each participant performed a total of nine $8 \mathrm{~s}$ sprint tests under different experimental conditions (Figure 1). Each participant performed these sprint tests in random order to avoid training and/or fatigue effects during the experiment.

A full recovery period of at least 5 min was imposed between each sprint; during this recovery period, the experimenter adjusted the handcycle. Participants were not given any recommendations concerning the propulsion techniques that could be used for the tests.

\section{Instrumentation}

An adjustable sports handcycle (Sopur Spirit 470, Sunrise Medical; Heidelberg, Germany) was used in this study. The handcycle's backrest was either tilted at different angles (B45, B65) or removed (WB). According to Mossberg et al.'s study on correcting differences in subject height, we adjusted the seat position relative to the backrest to obtain the same elbow extension $\left(15^{\circ}-20^{\circ}\right)$ for each participant and prevent complete elbow extension during maximal reach [16]. The handgrips were in a neutral position. The length of the standard-type cranks was $18 \mathrm{~cm}$. For all tests, tire pressure was set at 8 bars. 


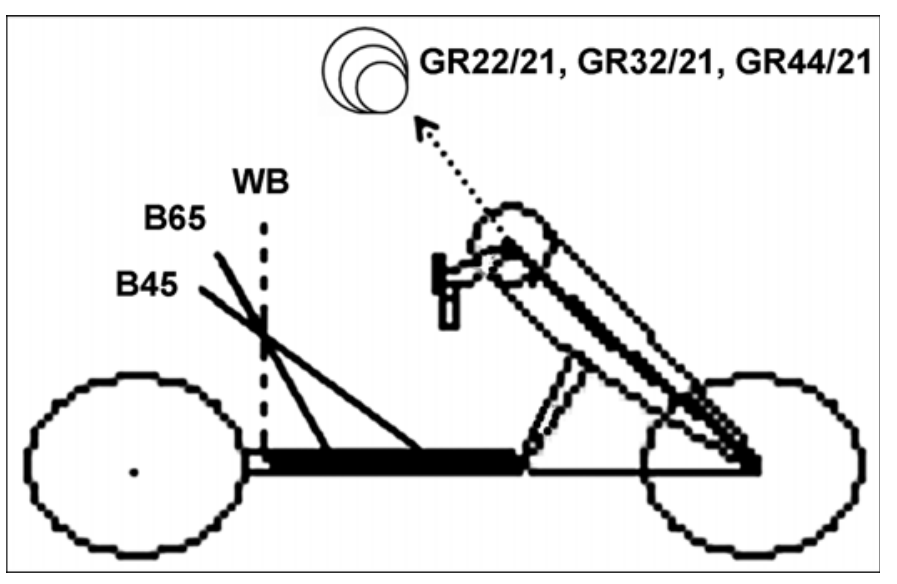

Figure 1.

Handcycle backrest positions $\left(\mathrm{B} 45=\right.$ backrest angle $45^{\circ}-50^{\circ}$ to horizontal, B65 = backrest angle $65^{\circ}-70^{\circ}$ to horizontal, $\mathrm{WB}=$ without backrest) and gear ratios (GRs) (GR22/21 = low, GR32/21 = medium, GR44/21 = high) used in study.

The handcycle was attached to a computer-linked ergocycle (Axiom; Elite, Fontaniva, Italy) equipped with a motor unit that imposes a constant rolling resistance on the handcycle's front wheel. Calibration of the Elite Axiom motor unit is not required. The rolling resistance is represented by a virtual slope that we set at 1 percent using the Elite Axiom software.

A three-dimensional movement analysis was performed with a Vicon 370 system (Oxford Metrics; Oxford, United Kingdom) with a sample rate of $60 \mathrm{~Hz}$. This measurement system comprises six digital cameras equipped with an infrared flash system. Marker locations are similar to Roux et al.'s protocol [17]. We placed 22 anatomical and technical markers on the participants (anatomical markers: one each on the forehead, chin, center of the sternum, seventh cervical [C7] vertebra, and third lumbar [L3] vertebra; two each on the acromions, temples, styloid process of the radius and cubitus, and metacarpals of the auricular and index fingers; technical markers: four markers on the arm and three on the forearm). The backrest was modified so that the L3 marker was visible. The reflective markers were spherical: $25 \mathrm{~mm}$ for the anatomical markers and $9 \mathrm{~mm}$ for the technical markers. The technical markers allowed us to use Roux et al.'s global optimization method, significantly minimizing measurement errors due to sliding of the skin [17].

\section{Data Measurement}

Before the beginning of each test, the capture volume was calibrated. In the absence of overall movement (and thus skin movement), we used a static trial to measure the position of the anatomical markers and to determine the joints' respective centers of rotation. Based on the static trial, anatomical frames were defined according to the International Society of Biomechanics' guidelines [18]. According to Schmidt et al.'s method, the shoulder center is assumed to be $7 \mathrm{~cm}$ inferior to the acromion marker, which is the average distance measured with a ruler [19]. The elbow joint center is the middle between the medial and lateral elbow markers. The joint center of the wrist is the middle between the ulnar and radial wrist markers. The upper part of the human body was considered an articulated system containing rigid bodies corresponding to the following body segments: head, trunk, arm, forearm, and hand. The trunk was characterized by the C7, L3, and sternum markers. We chose Euler angles to describe the relative movement of the body segments and used a global optimization method to minimize measurement errors due to skin movement [17]. Kinematic data were filtered by a fourth-order digital Butterworth $^{*}$ filter with a cutoff frequency of $6 \mathrm{~Hz}$ [20].

For each sprint, we calculated the average velocity (kilometers per hour), the cycle frequency (revolutions per minute), the maximum and minimum angles and total range of motion $(\mathrm{ROM})\left(^{\circ}\right)$ of the $\mathrm{F} / \mathrm{E}$, adduction/abduction $(\mathrm{Ad} / \mathrm{Ab})$, shoulder internal/external rotation (Ri/Re), and elbow and trunk F/E. Data were recorded throughout the trials, but we only accounted for three consecutive cycles of each $8 \mathrm{~s}$ sprint when the velocity had reached a plateau (thus allowing more reliable calculation of the average velocity). In Figure 2, one can observe that, from the start of the sprint, the velocity curve increases, reaches average maximal velocity during the first $4 \mathrm{~s}$, and stabilizes during the last $4 \mathrm{~s}$. Between the two arrows, three consecutive cycles are presented.

\section{Statistical Analysis}

We used SigmaStat (Systat Software Inc; San Jose, California) and MATLAB (The MathWorks; Natick, Massachusetts) software to perform all statistical tests and included the mean and SD values calculated from the last three consecutive cycles from each experimental trial. A two-factor analysis of variance (ANOVA) for repeated measures with a $3 \times 3$ design (GR22/21, GR32/21, and GR44/21 and B45, B65, and WB) was applied to

\footnotetext{
*The Butterworth filter (also called a "maximally flat magnitude" filter) is one type of electronic filter design. It is designed to have a frequency response that is as flat as mathematically possible in the passband.
} 


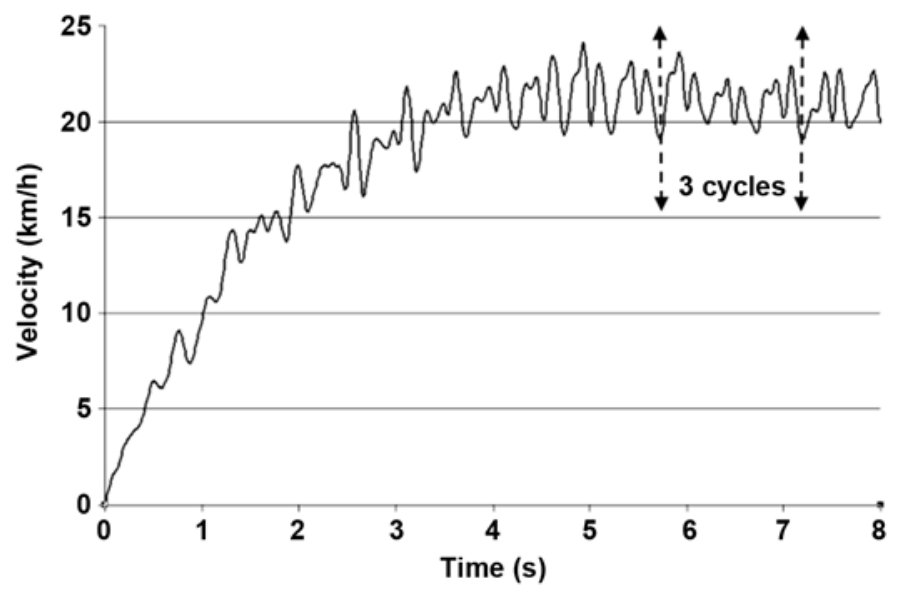

Figure 2.

Representative velocity variation obtained for one participant during 8 s sprint on handcycle.

determine the effect of backrest position and crank mode on the kinematic parameters. Significant statistical analyses were followed by a Bonferroni post hoc test. The significance threshold was set at $p<0.05$.

\section{RESULTS}

The Table shows the quantitative results for the kinematic data (mean $\pm \mathrm{SD}$ ) from each of the nine test conditions. The two-factor ANOVA for repeated measures showed statistically significant effects of backrest position and GR on velocity $(p<0.001)$ and crank frequency $(p<0.001)$, without any interaction between these two factors. Indeed, the velocity rose and the crank frequency dropped as the GR increased. For the different backrest positions and GRs, we also observed a significant difference in trunk $\mathrm{F} / \mathrm{E}(p<0.001)$ and a significant interaction between these two parameters. Concerning the joint ROM, we observed an influence of GR $(p<0.05)$ on shoulder $\mathrm{Ad} / \mathrm{Ab}$ and $\mathrm{Ri} / \mathrm{Re}$ and an influence of backrest position $(p<0.05)$ on elbow $\mathrm{F} / \mathrm{E}$ and shoulder Ri/Re.

For ease of reading, the Table does not show the results obtained with the Bonferroni post hoc test in the different test conditions. The results of Bonferroni post hoc tests concerning trunk $\mathrm{F} / \mathrm{E}$ and velocity are shown in Figure 3. Figure 3 represents both the maximal velocity (kilometers per hour) and the maximal amplitude of the trunk F/E in each of the nine test conditions. Significant differences $(p<0.001)$ were observed for the backrest position factor (B45 vs WB and B65 vs WB). Furthermore, to check for a correlation between trunk F/E and the participants' maximal velocity, we performed a Spearman correlation test. The resulting coefficient was 0.4 , which means that the two variables were not significantly correlated. Thus, the statistical results (the Bonferroni post hoc tests) shown in Figure 3 only indicate that greater trunk $\mathrm{F} / \mathrm{E}$ and greater maximal velocity were achieved without a backrest.

Table.

Mean \pm standard deviation for kinematic parameters measured during nine handcycle conditions that combined three backrest positions (B45 $=$ backrest angle $45^{\circ}-50^{\circ}$ to horizontal, B65 = backrest angle $65^{\circ}-70^{\circ}$ to horizontal, and $\mathrm{WB}=$ without backrest) and three gear ratios (GRs) (GR22/21 = low, GR32/21 = medium, and GR44/21 = high).

\begin{tabular}{|c|c|c|c|c|c|c|c|c|c|c|c|c|}
\hline \multirow{2}{*}{ Parameter } & \multicolumn{3}{|c|}{ B45 } & \multicolumn{3}{|c|}{ B65 } & \multicolumn{3}{|c|}{ WB } & \multicolumn{3}{|c|}{ ANOVA } \\
\hline & GR22/21 & GR32/21 & GR44/21 & GR22/21 & GR32/21 & GR44/21 & GR22/21 & GR32/21 & GR44/21 & Back & GR & Int \\
\hline Velocity (km/h) & $17.3 \pm 3.0$ & $22.7 \pm 4.2$ & $26.8 \pm 7.2$ & $17.6 \pm 2.6$ & $23.3 \pm 4.5$ & $26.4 \pm 6.3$ & $18.8 \pm 3.2$ & $25.7 \pm 4.9$ & $30.2 \pm 8.6$ & * & * & NS \\
\hline \multicolumn{13}{|l|}{ Angle $\left(^{\circ}\right)$} \\
\hline Trunk F/E & $4.9 \pm 2.0$ & $6.7 \pm 3.0$ & $10.0 \pm 4.6$ & $4.9 \pm 1.8$ & $6.1 \pm 4.1$ & $7.6 \pm 2.4$ & $9.1 \pm 3.5$ & $10.8 \pm 4.5$ & $20.7 \pm 10.5$ & * & * & * \\
\hline \multicolumn{13}{|l|}{ Shoulder } \\
\hline $\mathrm{Ab} / \mathrm{Ad}$ & $25.8 \pm 11.0$ & $28.2 \pm 9.5$ & $29.7 \pm 10.9$ & $25.4 \pm 6.2$ & $28.3 \pm 7.9$ & $30.9 \pm 8.0$ & $32.1 \pm 12.7$ & $30.7 \pm 8.4$ & $35.4 \pm 10.4$ & 0.057 & $\dagger$ & NS \\
\hline $\mathrm{Ri} / \mathrm{Re}$ & $36.8 \pm 8.8$ & $31.5 \pm 14.1$ & $34.9 \pm 19.0$ & $45.2 \pm 15.3$ & $29.3 \pm 9.0$ & $26.5 \pm 11.8$ & $59.1 \pm 18.6$ & $41.5 \pm 15.5$ & $37.7 \pm 13.8$ & $\dagger$ & $\dagger$ & NS \\
\hline Elbow F/E & $75.1 \pm 7.7$ & $75.3 \pm 6.8$ & $75.5 \pm 8.9$ & $77.7 \pm 11.1$ & $74.3 \pm 10.3$ & $72.5 \pm 11.4$ & $82.3 \pm 9.6$ & $79.1 \pm 9.4$ & $76.2 \pm 6.8$ & $\dagger$ & NS & NS \\
\hline
\end{tabular}
${ }^{*} p<0.001$. ${ }^{\dagger} p<0.05$.

$\mathrm{Ad} / \mathrm{Ab}=$ adduction/abduction, ANOVA $=$ analysis of variance, $\mathrm{F} / \mathrm{E}=$ flexion/extension, Int $=$ Interaction (between backrest [back] and GR), NS $=$ nonsignificant (differences), $\mathrm{Ri} / \mathrm{Re}=$ internal/external rotation. 


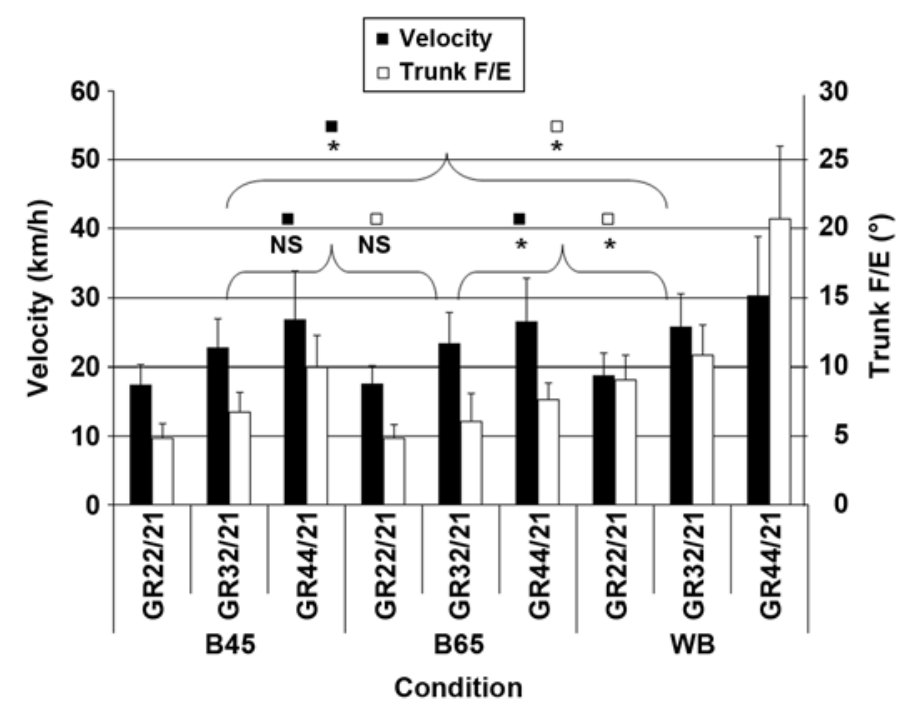

Figure 3.

Bonferroni post hoc test results, showing maximal velocity and trunk flexion/extension (F/E) amplitudes for nine test conditions that combined three backrest positions (B45 $=$ backrest angle $45^{\circ}-50^{\circ}$ to horizontal, B65 = backrest angle $65^{\circ}-70^{\circ}$ to horizontal, $\mathrm{WB}=$ without backrest) and three gear ratios (GRs) (GR22/21 = low, GR32/21 = medium, and GR44/21 = high). ${ }^{*} p<0.001$. NS = nonsignificant.

\section{DISCUSSION}

This study assessed the effect of backrest positions (both alone and in combination with different GRs) on nondisabled subjects' kinematics during handcycling sprinting. The major finding of this study is that in the absence of a backrest, more trunk movement is observed and allows greater velocity; this finding was especially true for higher GRs (Table and Figure 3).

\section{Methods}

None of the study participants had any previous experience in handcycling. Consequently, our findings represent a potential shortcoming in that they may not be completely transferable to wheelchair-dependent users with trunk and/or upper-limb disabilities. Future studies with different subjects (especially those with limited trunk function) are still required. Nonetheless, our subjects' lack of experience and training in this task meant that we avoided training effects related to the propulsion modes and backrest position used by experienced handcyclers. Studies of the effects of ergonomic adjustments and design within nonexperienced subjects have already demonstrated interest in arm crank exercise [16,21-22] and in handcycling [12,14]. The effects of backrest adjustment were fully investigated in our experiment, since we excluded the potential confounding factor of limited or variable trunk function by using nondisabled subjects. Indeed, nondisabled subjects have been shown to generate consistent results in studies investigating wheelchair ergonomic variations [23]. Consequently, the present study was performed with a homogenous subject group with an equivalent lack of experience. Moreover, the boom in handcycling has meant that this sport has spread to a broader population. Handcycling competitions are not only for people with limited trunk function (such as those with spinal cord injuries [SCIs]) but are also open to athletes with disabilities who have good trunk control (such as athletes with poliomyelitis or leg amputations). Furthermore, outside International Paralympic Committee events, the European Handcycle Circuit includes nondisabled athletes in its championships [24]; this further boosts the sport's development and increases interest in handcycling for the nondisabled. Hence, this study was well controlled, had an original setup, and offers good indications about the efficiency of handbiking for novice nondisabled subjects.

Another drawback of this study is that the athletes' power outputs were unknown, because of technical limitations. The ergocycle is equipped with a motor unit that imposes a constant rolling resistance on the front wheel, but the Elite Axiom tends to overestimate the average power [25-26], so the value of power output was not used in this study.

Lastly the results of this study cannot be generalized to steady state handcycling since our participants performed maximal sprints for $8 \mathrm{~s}$. The specific movement dynamics in handcycling can be defined as starting, accelerating, steady state wheeling, and sprinting. Even if handcyclers use steady state wheeling most, the ability to accelerate quickly from a standstill is also important in handcycling. Furthermore, according to Vanlandewijck et al., "trunk motion might be one of the most important force-generating mechanisms during high resistance wheeling, such as accelerating from standstill or sprinting” [5]. Indeed, the $8 \mathrm{~s}$ maximal sprint paradigm has already demonstrated its value in handcycling [9] and wheelchair propulsion studies [27-28]. In these latter investigations, data were recorded for three or five consecutive cycles of each $8 \mathrm{~s}$ sprint when the velocity had reached a plateau. In Hintzy et al.'s study, the results (obtained from 17 females who performed nine maximal 
$8 \mathrm{~s}$ wheelchair sprints with friction torques varying from 0-4 N) showed that the maximal velocity was attained after two to three arm cycles and remained constant during the rest of the sprint [28]. Likewise, during $8 \mathrm{~s}$ handcycling sprints, we and others demonstrated that the velocity curve increases rapidly, climbs to reach the mean maximal velocity during the first few seconds, and then stabilizes during the last few seconds [27].

\section{Backrest Positions}

We paid particular attention to the trunk F/E maximal amplitudes and velocities according to both the backrest positions (B45, B65, WB) and GRs (GR22/21, GR32/21, GR44/21) used. We observed significant differences as a function of backrest adjustment (Table, Figure 3), principally when we compared WB with B45 and B65. Moreover, these differences were accentuated as the GR increased. Indeed, trunk F/E was the highest for GR44/21, with amplitudes of $10.0^{\circ} \pm 4.6^{\circ}$ for B $45,7.6^{\circ} \pm 2.4^{\circ}$ for B65, and $20.7^{\circ} \pm 10.5^{\circ}$ for WB. Hence, in the absence of a backrest, we noticed a simultaneous trunk F/E increase and higher maximal velocity for a higher GR (Figure 3), thus indicating a performance improvement-in terms of average maximal velocity-without any interaction between these two factors (Table). Moreover, we also highlighted that backrest positions influence other parameters, in particular, the amplitude of joint angles in general and elbow $\mathrm{F} / \mathrm{E}$ and shoulder Ri/Re. Indeed, these joint amplitudes were greatest in the absence of a backrest (WB) and with the lowest GR (GR22/21). One might suggest that these angle variations were due to an accompaniment of trunk motion. However, we did not find any correlation between velocity and trunk $\mathrm{F} / \mathrm{E}$, which does not enable us to conclude that participants with the largest trunk F/E reached the highest propulsive velocity, or vice versa. Nevertheless, our results agree with van Breukelen's theoretical classification [15] in which propulsion without a backrest was suggested to be equivalent to ATP propulsion, thus allowing athletes to actively use their trunk muscles during the propulsive phase and, as a result, improve movement efficiency. AP propulsion is best characterized by a backrest tilted backward, a seat set close to the arm-pedaling system, and a shoulder overhang by the crank axis. Thus, in contrast to ATP propulsion, the athlete essentially stays in contact with the backrest and the trunk is not as involved in force production. Propulsion is performed with the arms, and trunk movement is impossible because of the semirecumbent position. However, AP propulsion does mean that the handcycler achieves better stability.

\section{Gear Ratio}

In addition to our observed backrest position effects on angle parameters (and especially trunk $\mathrm{F} / \mathrm{E}$ ), we also detected a GR effect. The results in the Table indicate greater trunk F/E for the highest GR, whatever the backrest adjustment, which agrees with a previous kinematic study that investigated arm and trunk ROM during handcycling [10]. We found greater trunk F/E ROM for the highest GR and explained this phenomenon by either greater resistance on the cranks (because of the high GR, causing the back and the backrest to separate during the pull phase) or by the athletes' use of trunk inertia (by tilting their trunk forward to improve propulsion) [9].

With respect to the other angle parameters, GR also influenced amplitude variation. We observed significantly higher shoulder $\mathrm{Ad} / \mathrm{Ab}$ amplitudes at a higher GR. In contrast to the hand rim wheelchair, handcycling has no "air phase" (that is, a period during which the hands are not in contact with the hand rims) and propulsion is performed in a closed loop. Consequently, shoulder $\mathrm{Ad} / \mathrm{Ab}$ is one of the few degrees of freedom that can be modified during handcycling (if the back is pressed against the backrest) by raising of the elbow.

GR not only influences trunk motion but also affects crank frequency and propulsion velocity. Hence, whatever the backrest position, we noticed an increase in the average maximal velocity and a decrease in crank frequency at a higher GR. These results, confirmed by the Bonferroni post hoc test, demonstrate the performance advantage of a higher GR and, indeed, confirm and extend the findings of a previous kinematic study [9]. However, discrepancies exist with other studies that reported better mechanical efficiency at a lower GR [12]. But, van der Woude et al. performed testing at a submaximal intensity and with low crank frequency (44 rpm), which prompted the authors to suggest the need for further investigations at higher crank frequencies (given the fact that athletes can exceed $50 \mathrm{~km} / \mathrm{h}$ with very high crank rates) [12]. Indeed, in our investigation of sprint conditions by nondisabled subjects, we used a high crank frequency (108-153 rpm). According to Faupin et al., we can conclude that the highest GR helps the user obtain a higher maximal velocity during a sprint while it reduces the frequency of movements [9]. According to Vanlandewijck et al.'s review, handcycling with a gearing system offers evident power transmission advantages 
compared with hand rim wheelchair propulsion [5]. If the traditional hand rim racer is classified in terms of athletics, handcycles are closer to cycling because their users can select and maintain a desired cadence via GR.

\section{CONCLUSIONS}

From this study of novice nondisabled subjects performing $8 \mathrm{~s}$ handcycling sprints, one can conclude that performance improves in the absence of a backrest. In fact, for this position, we noticed a simultaneous increase in trunk $\mathrm{F} / \mathrm{E}$ and maximal velocity for a higher GR. These results agree with the theoretical van Breukelen classification in which the ATP type of propulsion allows the athletes to actively use their trunk muscles during the propulsive phase [15]. However, only persons with good trunk stability can use a handcycle without a backrest. Conversely, athletes with SCI (i.e., with lower inherent trunk stability) who compete in handcycling events generally tilt the backrest backward (at around $45^{\circ}$ to the horizontal). Clearly, future experiments need to be performed in these specific groups with SCI.

\section{ACKNOWLEDGMENTS}

We thank Olivier Rémy-Néris for scientific help and Stéphane Bouilland for technical assistance during the experiment. We also thank the Institut Garches (Hôpital Raymond Poincaré, 104, boulevard Raymond-Poincaré, 92380 Garches), which allowed us to perform this project.

Portions of this work were presented at the 3rd International Congress Restoration of (Wheeled) Mobility in SCI Rehabilitation: State of the Art III, Vrije Universiteit, Amsterdam, the Netherlands, April 19-21, 2004.

This material is the result of work supported with resources and facilities of the J. Calvé center (Groupe Hopale, 72 esplanade Parmentier, 62608 Berck sur mer).

The authors have declared that no competing interests exist.

\section{REFERENCES}

1. Mukherjee G, Samanta A. Physiological response to the ambulatory performance of hand-rim and arm-crank propulsion systems. J Rehabil Res Dev. 2001;38(4):391-99. [PMID: 11563492]
2. Dallmeijer AJ, Zentgraaff ID, Zijp NI, Van der Woude LH. Submaximal physical strain and peak performance in handcycling versus handrim wheelchair propulsion. Spinal Cord. 2004;42(2):91-98. [PMID: 14765141]

3. Janssen TW, Dallmeijer AJ, Van der Woude LH. Physical capacity and race performance of handcycle users. J Rehabil Res Dev. 2001;38(1):33-40. [PMID: 11322469]

4. Abel T, Kröner M, Rojas Vega S, Peters C, Klose C, Platen $\mathrm{P}$. Energy expenditure in wheelchair racing and handbiking-A basis for prevention of cardiovascular diseases in those with disabilities. Eur J Cardiovasc Prev Rehabil. 2003; 10(5):371-76. [PMID: 14663299]

5. Vanlandewijck Y, Theisen D, Daly D. Wheelchair propulsion biomechanics: Implications for wheelchair sports. Sports Med. 2001;31(5):339-67. [PMID: 11347685]

6. Van der Woude LH, Dallmeijer AJ, Janssen TW, Veeger D. Alternative modes of manual wheelchair ambulation: An overview. Am J Phys Med Rehabil. 2001;80(10):765-77.

[PMID: 11562560]

7. Van der Woude LH, Veeger HE, Dallmeijer AJ, Janssen TW, Rozendaal LA. Biomechanics and physiology in active manual wheelchair propulsion. Med Eng Phys. 2001; 23(10):713-33. [PMID: 11801413]

8. Van der Woude LH, De Groot S, Janssen TW. Manual wheelchairs: Research and innovation in rehabilitation, sports, daily life and health. Med Eng Phys. 2006;28(9): 905-15. [PMID: 16504565]

9. Faupin A, Gorce P, Campillo P, Thevenon A, Rémy-Néris O. Kinematic analysis of handbike propulsion in various gear ratios: Implications for joint pain. Clin Biomech (Bristol, Avon). 2006;21(6):560-66. [PMID: 16510220]

10. Verellen J, Gers B, Van de Vliet P, Vanlandewijck Y. Consistency of the within cycle torque distribution pattern during hand cycling: A pilot study. Eur Bull Adapted Phys Activ. 2004;3(2). Available from: http://www.bulletin-apa.com

11. DeCoster A, Van Laere M, Blonde W. Electromyographic activity of shoulder girdle muscles during handbiking. In: Van der Woude LH, Hopman MT, Van Kemenade CH, editors. Biomedical aspects of manual wheelchair propulsion: The state of the art II. Washington (DC): IOS Press; 1999. p. 138-41.

12. Van der Woude LH, Bosmans I, Bervoets B, Veeger HE. Handcycling: Different modes and gear ratios. J Med Eng Technol. 2000;24(6):242-49. [PMID: 11315650]

13. Abel T, Vega S, Bleicher I, Platen P. Handbiking: Physiological responses to synchronous and asynchronous crank montage. Eur J Sport Sci. 2003;3(4):1-9.

14. Dallmeijer AJ, Ottjes L, De Waardt E, Van der Woude LH. A physiological comparison of synchronous and asynchronous hand cycling. Int J Sports Med. 2004;25(8):622-26. [PMID: 15532007] 
15. Van Breukelen K. Handbikes: Arm power versus arm trunk power, an ergonomic classification of handbikes. Armpowered by Double Performance. 2001. Available from: http://www.handbike.de/ftp/downloads2002/arm-vs-trunk.pdf

16. Mossberg K, Willman C, Topor MA, Crook H, Patak S. Comparison of asynchronous versus synchronous arm crank ergometry. Spinal Cord. 1999;37(8):569-74. [PMID: 10455533]

17. Roux E, Bouilland S, Godillon-Maquinghen AP, Bouttens D. Evaluation of the global optimisation method within the upper limb kinematics analysis. J Biomech. 2002;35(9): 1279-83. [PMID: 12163317$]$

18. Wu G, Cavanagh PR. ISB recommendations for standardization in the reporting of kinematic data. J Biomech. 1995; 28(10):1257-61. [PMID: 8550644]

19. Schmidt R, Disselhorst-Klug C, Silny J, Rau G. A markerbased measurement procedure for unconstrained wrist and elbow motions. J Biomech. 1999;32(6):615-21.

[PMID: 10332626]

20. Cooper RA, DiGiovine CP, Boninger ML, Shimada SD, Koontz AM, Baldwin MA. Filter frequency selection for manual wheelchair biomechanics. J Rehabil Res Dev. 2002; 39(3):323-36. [PMID: 12173753]

21. Hopman MT, Van Teeffelen WM, Brouwer J, Houtman S, Binkhorst RA. Physiological responses to asynchronous and synchronous arm-cranking exercise. Eur J Appl Physiol Occup Physiol. 1995;72(1-2):111-14. [PMID: 8789580]

22. Powers SK, Beadle RE, Mangum M. Exercise efficiency during arm ergometry: Effects of speed and work rate. J Appl Physiol. 1984;56(2):495-99. [PMID: 6706760]
23. Van der Woude LH, Formanoy M, De Groot S. Hand rim configuration: Effects on physical strain and technique in unimpaired subjects? Med Eng Phys. 2003;25(9):765-74. [PMID: 14519349]

24. European Handcycling Federation. EHC Rulebook 2007. Available from: http://www.european-handbike.de/EHC/ ehc regeln/EHF Rulebook 2007.pdf

25. Bertucci W, Duc S, Villerius V, Grappe F. Validity and reliability of the Axiom PowerTrain cycle ergometer when compared with an SRM powermeter. Int J Sports Med. 2005;26(1):59-65. [PMID: 15643536]

26. Nesi X, Bosquet L, Carter H, Dekerle J, Pelayo P. The validity of cycling power recorded by the Elite Axiom Ergometer. J Hum Mov Stud. 2006;50:91-102.

27. Faupin A, Campillo P, Weissland T, Gorce P, Thevenon A. The effects of rear-wheel camber on the mechanical parameters produced during the wheelchair sprinting of handibasketball athletes. J Rehabil Res Dev. 2004;41(3B):421-28. [PMID: 15543460]

28. Hintzy F, Tordi N, Predine E, Rouillon JD, Belli A. Forcevelocity characteristics of upper limb extension during maximal wheelchair sprinting performed by healthy ablebodied females. J Sports Sci. 2003;21(11):921-26.

[PMID: 14626371]

Submitted for publication October 30, 2006. Accepted in revised form June 26, 2007. 